\title{
Methicillin-resistant Staphylococcus aureus Hand Infections at UP-PGH: A Retrospective Study from 2005-2010
}

\author{
Jose Ma. D. Bautista ${ }^{1}$ and Carl Ryan Marino D. Taguba ${ }^{2}$ \\ ${ }^{1}$ Department of Orthopedics, College of Medicine and Philippine General Hospital, University of the Philippines Manila \\ 2Department of Orthopedics, East Avenue Medical Center, Quezon City, Philippines
}

\begin{abstract}
Objectives. To determine the incidence of MRSA among admitted hand patients at UP-PGH from 2005 to 2010 and determine the antibiotic susceptibility pattern.

Methods. Records of admitted hand patients of all ages were identified and wound culture results were reviewed. A total of 482 wound cultures from the 5 -year period were examined.

Results and Conclusion. From an initial decreasing trend, there was a sharp increase in incidence of MRSA hand infections from $2008(11.8 \%)$ to 2009 (22.5\%). Vancomycin had the highest number of susceptible cases from 2005 to 2008, but dropped quite sharply to the least number of susceptible cases in 2010. Chloramphenicol and erythromycin had the highest number of susceptible MRSA hand cases in recent years.
\end{abstract}

Key Words: hand, infections, Philippine General Hospital, MRSA, community-acquired

\section{Introduction}

Infections of the hand can result in profound morbidity, including stiffness, contracture, and amputation, if not recognized early and given the appropriate antibiotics., ${ }^{1,2,3}$ Factors that influence the outcome of infection include location of infection, causative organism, timing of treatment, adequacy of surgical drainage, efficacy of antibiotic therapy, health status and immunocompetence of the infected person. ${ }^{1,4,5}$ The most common organism isolated from hand and other soft tissue infections is Staphylococcus aureus which thrive as human skin flora. ${ }^{1,3}$ First-generation cephalosphorins have been traditionally the cornerstone antibiotic treatment for acute hand infections. ${ }^{6}$

Methicillin-resistant Staphylococcus aureus (MRSA) was first described in 1961, a short period after the introduction of methicillin in 1959.6,7 Most reported cases were hospital

Corresponding author: Jose Ma. D. Bautista, MD

Department of Orthopedics

Philippine General Hospital

University of the Philippines Manila

Taft Avenue, Ermita, Manila 1000 Philippines

Telephone: +6325548466

Fax No:. +6325218538

Email: jdbautista2@up.edu.ph acquired (HA-MRSA). ${ }^{1,6-8}$ Established risk factors for MRSA infection include recent hospitalization or surgery, residence in a long-term care facility, dialysis, and indwelling percutaneous medical devices and catheters. , $^{1,6}$

By the mid-1990s, however, younger and otherwise healthy individuals were acquiring MRSA infections despite not having contact with the hospital environment. ${ }^{1,6}$ This has started the concept of community-acquired methicillinresistant Staphylococcus aureus (CA-MRSA) infections. This type of infection has been associated with a high incidence in at-risk populations and settings, including extremes of age, contact sports, shared athletic equipment, immunosupression, prisons, daycare centers, households, schools, and athletic or military facilities. ${ }^{6,8}$ In recent years, however, CA-MRSA has been shown to have increasing prevalence in soft tissue infections. In particular, CA-MRSA infections of the hand have been increasing since the first case reports in 2000 have been described. ${ }^{1-9}$

At the researcher's institute, a study done by the Hospital Infection Control Unit (HICU) and Bacteriology Section showed that the overall MRSA incidence is $51 \%$ in 2009 from $50 \%$ in $2008 .{ }^{10}$ The incidence of hand infections, however, was not specified.

The objectives of the present study were (1) to determine the incidence of MRSA among admitted hand patients at UP-PGH from 2005 to 2010; and (2) to determine the antibiotic susceptibility pattern. Specifically, (1) to determine the most common types of hand infections; (2) to determine the most common sites of involvement; and (3) to determine the most common location of involvement.

\section{Methods}

Cases of admitted hand patients of all ages from 2005 to 2010 at the researchers' institution were identified. Wound culture results of these patients were reviewed. Excluded from the study were non-MRSA patients and patients with prior culture-proven MRSA.

\section{Results}

A total of 1880 hand patients were admitted from year 2005 to 2010. Out of these, 482 had wound cultures done. Among those with wound cultures, 83 (17.2\%) were positive for MRSA. Sixty-two (75\%) were males with mean age of 
29.4 years (age range 1 to 64 years) and 21 (25\%) were females with mean age of 29.8 years (age range 1 to 60 years). Figure 1 shows the yearly number of hand cases with wound cultures with number of positive MRSA. The incidence of MRSA among admitted hand patients from 2005 to 2010 is shown in Figure 2. There was an increasing trend from 2005 to 2006 followed by a decrease in the next two years. There was a sharp increase, however, in 2009 (from 11.8 to $22.6 \%$ ) with minimal decrease in 2010 (20.5\%).

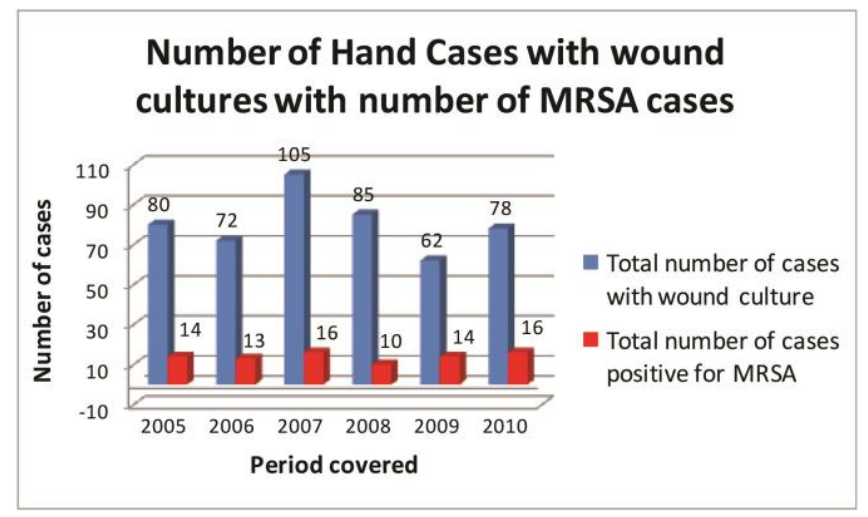

Figure 1. Number of hand cases with wound cultures per year with number of cases positive for MRSA.

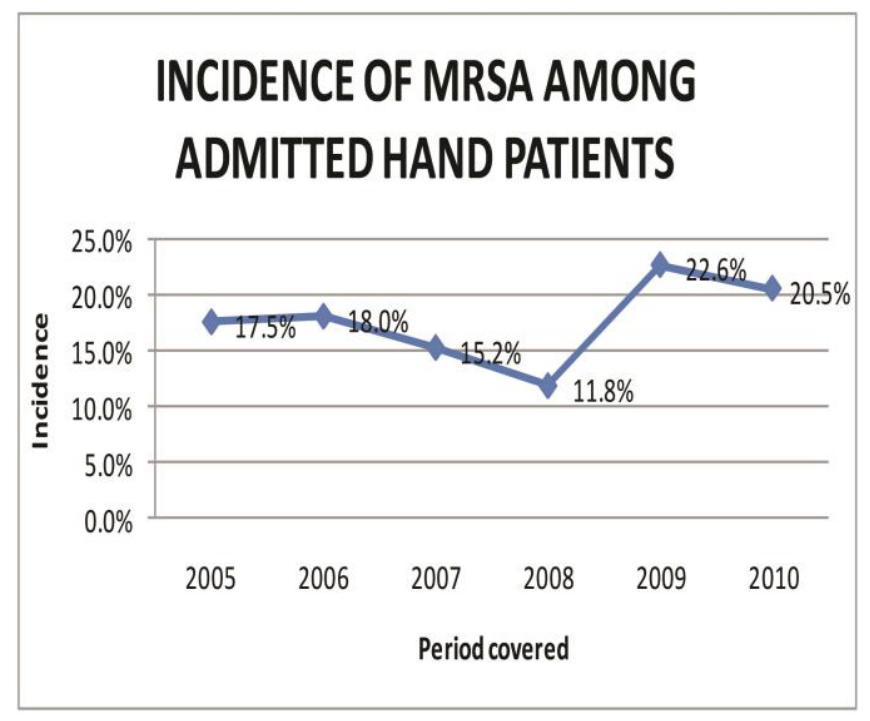

Figure 2. Incidence of MRSA Hand Infections from 2005 to 2010 among admitted patients at UP-PGH.

Among the types of hand infection, the most common was deep space infection $(22.9 \%)$, followed by flexor tenosynovitis (19.3\%), chronic osteomyelitis (13.2\%), and felon $(12 \%)$. It must be noted that one type occurred in conjuction with other types of hand infection (Figure 3 ). The most commonly involved site is the subcutaneous region (Figure 4). Again, one site may be in conjunction with others.

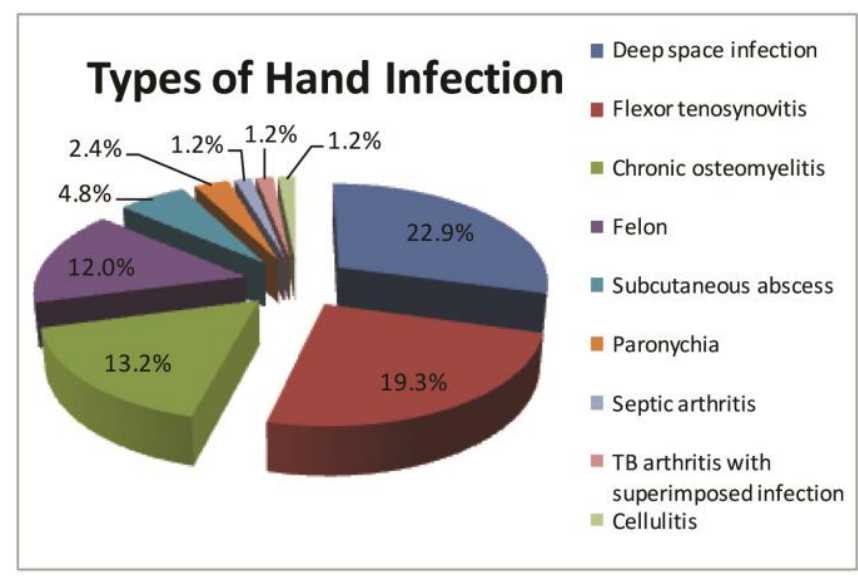

Figure 3. Types of hand infection seen in the study. Some appeared in conjunction with other types of infection.

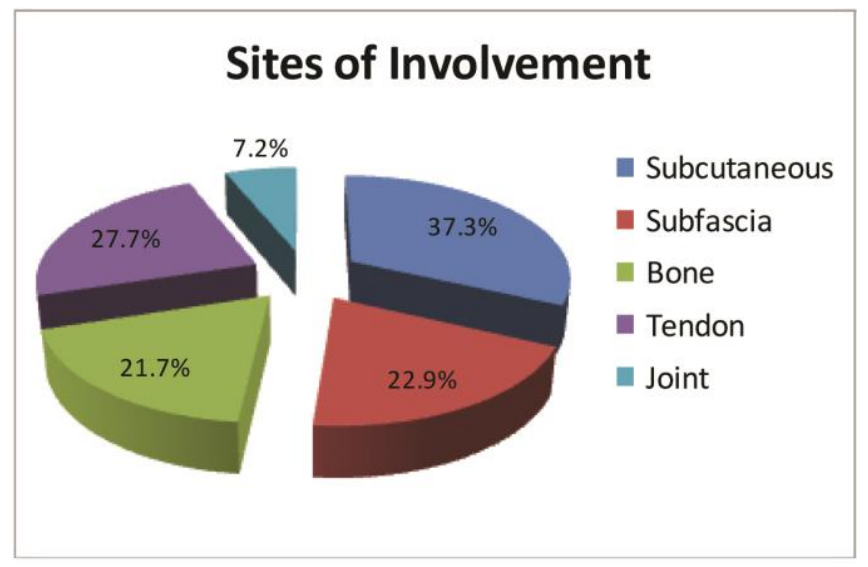

Figure 4. Sites of involvement of hand infections in the study. One type may be in concurrence with others.

Common locations of infection occurring in conjunction were the fingers $(26.5 \%)$, thumb $(25.3 \%)$, midpalmar space $(14.5 \%)$, thenar space $(4.8 \%)$, and hypothenar space $(4.8 \%)$. Among the 83 cases positive for MRSA, 63 (75.9\%) were of infectious (community-acquired) in origin. The rest were due to trauma (gunshot wound, hacking, crushing injury) (12 cases, $14.5 \%)$, surgical infection $(6,7.2 \%)$, blast injury (1, $1.2 \%)$, and burn injury $(1,1.2 \%)$.

The antibiotic susceptibility pattern of MRSA was also investigated. The top five antibiotics seen to be consistently sensitive to MRSA are vancomycin, erythromycin, chloramphenicol, trimethoprim-sulfamethoxazole, and clindamycin. The trend of each antibiotic from 2005 to 2010 is shown in Figure 5.

From 2005 to 2008, vancomycin had the highest number of susceptible cases of hand infections with MRSA. In 2009 and 2010, however, chloramphenicol and erythromycin had the most number of susceptible cases, respectively. Vancomycin's susceptibility decreased henceforth to fourth overall in 2009 and to eleventh in 2010. Erythromycin was 
fourth among the antibiotics tested in 2005, but was second to vancomycin from 2006 to 2008. It was third among all in 2009 but had the most number of susceptible cases in 2010. Chloramphenicol's susceptibility trend was increasing from 2005 to 2009, from being the sixth, fourth, and third twice in a row from 2005 to 2008, with the highest number of susceptible cases in 2009. It sharply dropped, however, (seventh among the antibiotics) the following year in 2010. Trimethoprim-sulfamethoxazole and clindamycin had similar trend from 2005 to 2010. Trimethoprimsulfamethoxazole alternated in the third to seventh spot overall while clindamycin was fourth to eighth among the antibiotics.

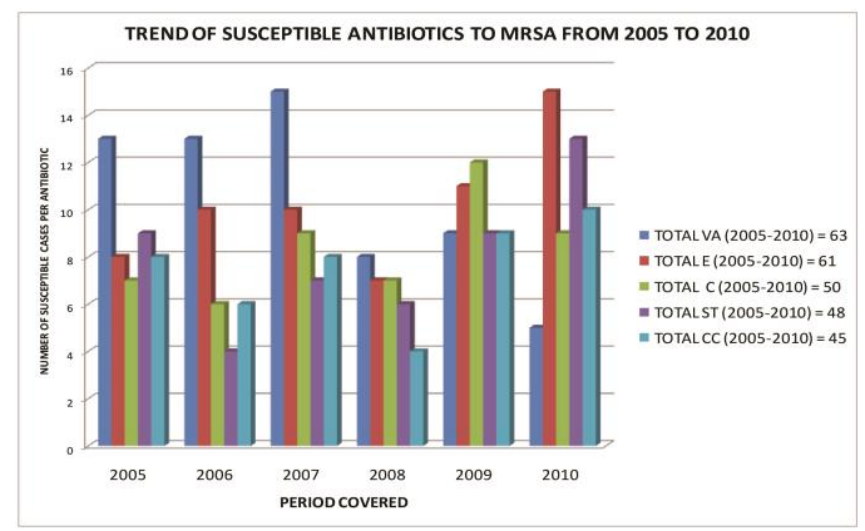

Figure 5. Trend of susceptibility of Vancomycin (VA), Erythromycin (E), Chloramphenicol (C), TrimethoprimSulfamethoxazole (ST), and Clindamycin (CC) to MRSA from 2005 to 2010.

\section{Discussion}

Several studies on MRSA hand infections have been done. O'Malley et al., in their retrospective review, had 85 culture-proven MRSA hand cases, 55 of which were men with mean age of 37 years. ${ }^{4}$ Downs et al. also had predominantly male cases with mean age of 39.1 years. ${ }^{3}$ At our institution, there was also male preponderance with a lower mean age (29.8 years). In O'Malley's study, there was a sharp increase in incidence of community-acquired MRSA from 2003 to 2005 (14 to 40\%) to 2007 (55\%). Downs et al. saw a $34 \%$ incidence among 110 cases of CA-MRSA in his 2year study (2003-2005). Bach et al. in $2005^{1}$ found a $73 \%$ incidence. In this 5-year retrospective study, there was a decrease in the trend from 2005 to 2008 until a sharp increase from 2008 to 2009. A local study done by the Hospital Infection Control Unit also showed an increased overall MRSA infection in the hospital in 2009. The most common mechanism of injury seen by $\mathrm{O}^{\prime}$ Malley were from lacerated wound, followed by puncture wounds and human or fight bite. In this study, most cases were infectious (communityacquired) in nature, followed by trauma (gunshot wound, hacking, and crushing injury), surgical infection, blast injury, and burn injury. With regard to risk of development of MRSA, though, there was no significant association between mechanism of injury and subsequent infection with MRSA according to $\mathrm{O}^{\prime}$ Malley and co-workers. ${ }^{4} \mathrm{~A}$ prospective study by Bach et al. in 2007 showed no association with age, gender, type of infection, location, co-morbidity. ${ }^{1}$

Intravenous vancomycin has been traditionally suggested by several authors to be given empirically for suspected cases of MRSA. ${ }^{1,4,6}$ Karanas et al., in their 2000 study, however, does not recommend an empiric coverage for MRSA because the indiscriminate use of IV Vancomycin will result in organisms resistant to the antibiotic. ${ }^{2}$ According to Tosti et al., ${ }^{6}$ IV Vancomycin is the drug of choice for in-patients with severe or refractory infection with systemic signs. Trimethoprim-sulfamethoxazole also has adequate coverage for MRSA and is relatively inexpensive. Clindamycin is a reasonable alternative although there is increasing resistance due to "inducible clindamycin resistance". Some S. aureaus isolates that appear erythromycin-resistant and clindamycin-susceptible by routine susceptible testing exhibit in vitro resistance to clindamycin during therapy. Other options for treatment for in-patients are linezolid, tigecycline, daptomycin, gentamycin, and chloramphenicol.1,4,6 Outpatient empiric treatment recommendations are oral clindamycin, trimethoprim-sulfamethoxazole, tetracyclines (doxycycline, minocycline), and rifampicin (in combination with other agents). ${ }^{4,9}$ The mainstay of treatment of MRSA according to Downs et al. is surgical debridement with antibiotic coverage based on culture sensitivities. ${ }^{3}$ The 2006 Summary of Experts' Meeting Convened by the Centers for Disease Control and Prevention recommends that local susceptibility data should be used to guide treatment. A beta-lactam agent (anti-staphylococcal penicillin or cephalosporin) is still a reasonable option for first line treatment in a patient with mild to moderate illness and no significant co-morbidities if the local prevalence of MRSA is low. A prevalence of greater than 10 to $15 \%$ has been suggested by some experts. ${ }^{9}$ The different antimicrobial agents mentioned above have advantages and disadvantages. The authors of the studies mentioned above agree that more data are needed from controlled clinical trials to establish optimal regimens for the treatment of MRSI skin and soft tissue infections.

In conclusion, this 5-year retrospective study shows that from an initial decreasing trend, there was a sharp increase in incidence of MRSA hand infections among admitted patients from $2008(11.8 \%)$ to $2009(22.6 \%)$. Vancomycin had the highest number of susceptible cases from 2005 to 2008, but this dropped quite sharply to the least number of susceptible cases in 2010. Chloramphenicol and erythromycin had the highest number of susceptible MRSA hand cases in recent years. 


\section{Limitations and recommendations}

The study design limits the authors' much-needed information. Records may be incomplete and inaccurate. This is a preliminary study to establish local incidence rates of MRSA specific for hand infections to guide the physician on rational use of antibiotics for empiric treatment. Future prospective studies will be valuable in further refining the profile of MRSA hand infections.

\section{References}

1. Bach HG, Steffin B, Chhadia AM, Kovachevich R, Gonzalez MH. Community-associated methicillin-resistant Staphylococcus aureus hand infections in an urban setting. J Hand Surg Am. 2007; 32(3):380-3.

2. Karanas YL, Bogdan MA, Chang J. Community-acquired methicillinresistant Staphylococcus aureus hand infections: case reports and clinical implications. J Hand Surg Am. 2000; 25(4):760-3.

3. Downs DJ, Wongworawat MD, Gregorius SF. Timeliness of appropriate antibiotics in hand infections. Clin Orthop Relat Res. 2007; 461:17-9.
4. O'Malley M, Fowler J, Ilyas AM. Community-acquired methicillinresistant Staphylococcus aureus infections of the hand: prevalence and timeliness of treatment. J Hand Surg Am. 2009; 34(3):504-8.

5. Ong YS, Levin LS. Hand infections. Plast Reconstr Surg. 2009; 124(4):225e-233e.

6. Tosti, R, Ilyas AM. Empiric antibiotics for acute infections of the hand. J Hand Surg Am. 2010; 35(1):125-8.

7. Pavlidakey PG. Newspath MRSA Update [Online]. 2009 [cited 2010 May]. Available from http:www.cap.orgs/apps/docs/newspat/0905/

8. Imahara SD, Friedrich JB. Community-acquired methicillin-resistant Staphylococcus aureus in surgically treated hand infections. J Hand Surg Am. 2010; 35(1):97-103.

9. Gorwitz RJ, Jernigan DB, Powers JH, Jernigan JA. Strategies for Clinical Management of MRSA in the Community: Summary of an Experts Meeting Convened by the Centers for Disease Control and Prevention [Online]. 2006 [cited 2010 May]. Available from http://www.cdc.gov/ncidod/dhqp/ar_mrsa_ca.html

10. Antibiotic Susceptibility Pattern: January-June 2009. Hospital Infection Control Unit (HICU) and Bacteriology Section, UP-PGH. 2010. Unpublished.

\section{Acta Medica Pfilippina \\ THE NATIONALHEALTH SCIENCE JOURNAL

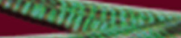

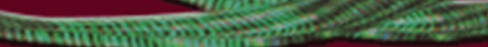

\section{Acta is now accepting} membership

Privileges of members:

- Allows you to submit articles for possible publication

- Have access to all the articles in the website (archives included) which can be downloaded and printed in pdf format

- Advertise your products/services in the available spaces of the website (for approval of the Editor-in-chief)

For details, please visit our website at www.actamedicaphilippina.com.ph or e-mail us at businessmanager@actamedicaphilippina.com.ph for any questions or queries. 